\title{
Mapping Pitch Representation in Neural Ensembles with fMRI
}

\author{
Timothy D. Griffiths ${ }^{1,2}$ and Deborah A. Hall ${ }^{3,4}$ \\ ${ }^{1}$ Institute of Neuroscience, Newcastle University Medical School, Newcastle upon Tyne, NE2 4HH, United Kingdom, ${ }^{2}$ Wellcome Trust Centre for \\ NeuroImaging, University College London, London WC1N 3BG, United Kingdom, ${ }^{3}$ National Institute for Health Research Nottingham Hearing Biomedical \\ Research Unit, Nottingham, NG1 5DU, United Kingdom, and ${ }^{4}$ School of Clinical Sciences, University of Nottingham, Queen's Medical Centre, Nottingham, \\ NG7 2UH, United Kingdom
}

Functional magnetic resonance imaging (fMRI) in humans and macaques allows a test of the hypothesis that there is a specialized neural ensemble for pitch within auditory cortex: a pitch center. fMRI measures the blood oxygenation level-dependent (BOLD) response related to regional synaptic activity (Logothetis et al., 2001). The distinction between synaptic activity and spike firing, and species differences encourage caution when comparing BOLD activity in humans and macaques to recordings from single neurons in ferret and marmoset in the previous mini-review. The BOLD data provide support for the pitch-center concept, with ongoing debate about its location.

\section{Introduction}

The auditory cortex of macaque and human is located in the superior temporal plane (Fig. 1). In the macaque, three core areas, including primary cortex (A1), run posterior to anterior. Using functional magnetic resonance imaging (fMRI), each can be seen to demonstrate distinct tonotopic (frequency) mapping (Petkov et al., 2006). Adjacent belt areas also show some degree of tonotopicity. In humans, organization of the auditory areas based on tonotopic mapping is currently debated (for recent human fMRI reports see Formisano et al., 2003; Talavage et al., 2004; Woods et al., 2009; Humphries et al., 2010; Da Costa et al., 2011; Langers and van Dijk, 2011). There is consensus that primary cortex is located in the medial part of the first transverse temporal gyrus (Heschl's gyrus, HG) in the superior temporal plane, while the status of lateral HG as a core or belt homolog is debated. Studies of human pitch representation have sought an area that is specialized for pitch coding within HG and adjacent auditory areas including the planum temporale (PT), posterior to $\mathrm{HG}$.

\section{fMRI approaches to pitch}

fMRI pitch studies have used subtraction methodology in which responses to a stimulus associated with pitch are compared with a control stimulus with no pitch. These experiments have sought to control for aspects of the different stimulus structure of the pitch and control sound to which fMRI might be sensitive, including the frequency composition (spectrum) of the sound (Oxenham, 2012). The first fMRI studies of cortical pitch responses used

Received April 4, 2012; revised July 18, 2012; accepted July 23, 2012.

T.D.G. is a Wellcome Trust Senior Clinical Fellow. All fMRI studies conducted by D.A.H. were supported by the Medical Research Council (UK). D.A.H. is currently supported by the National Institute for Health Research.

Correspondence should be addressed to either of the following: Timothy D Griffiths, Institute of Neuroscience, Newcastle University Medical School, Newcastle upon Tyne, NE2 4HH, UK, E-mail:t.d.griffiths@ncl.ac.uk, or Deborah A Hall, National Institute for Health Research Nottingham Hearing Biomedical Research Unit, Ropewalk House, 113 The Ropewalk, NG1 5DU, UK, E-mail: Deb.Hall@nottingham.ac.uk.

DOI:10.1523/JNEUROSCI.3813-12.2012

Copyright $\odot 2012$ the authors $\quad 0270-6474 / 12 / 3213343-05 \$ 15.00 / 0$ regular-interval noise (RIN), which is a type of noise to which the stimulus property of temporal regularity and the percept of pitch can be applied by a synthetic delay-and-add algorithm (Patterson et al., 2002; Hall et al., 2006). The use of low-pitch values, and a high-pass filter, minimizes the spectral ripple that occurs in the stimulus so that the stimulus can be compared with a control noise with the same passband to demonstrate responses that cannot be explained by changes in the time-averaged spectrum. The studies demonstrated maximal responses to RIN in lateral HG (although more medial responses in primary cortex are also observed; see Griffiths et al., 2010), which have been interpreted as pitch mappings. Similar activation in lateral HG has been demonstrated in an experiment in which a comparison was made between resolved harmonics (with high pitch salience) and unresolved harmonics (low pitch salience) in the same pass band (Penagos et al., 2004). Other studies have used forms of binaural pitch (Hall and Plack, 2007, 2009; Puschmann et al., 2010), in which the imposition of a phase shift between the ears in a particular pass band can be associated with a pitch. The experiments with binaural pitch have demonstrated responses in lateral HG and the adjacent part of PT.

These human studies suggest a regional specialization for pitch in the lateral superior temporal plane. The precise interpretation of the studies is critically dependent on differences between pitch and control stimuli other than the presence of pitch. For example, the modeled representation of RIN stimuli in the auditory pathway (Hall and Plack, 2009) demonstrates slow fluctuations in the spectrum over time and, perceptually, the creation of RIN from noise produces timbral as well as pitch change. Experiments using a more refined type of control noise containing similar fluctuations did not show differences in RIN-related activation in lateral HG that are significant (Barker et al., 2012). Experiments using resolved and unresolved harmonics produce differences in the auditory spectrum as well as differences in the pitch percept. The experimental manipulations to produce binaural pitch also cause a difference in the perceived spatial location 
Macaque

A

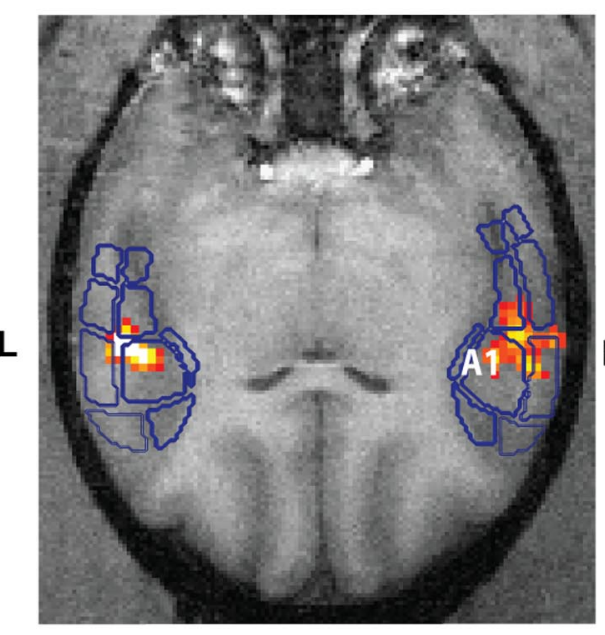

$\mathbf{P}$

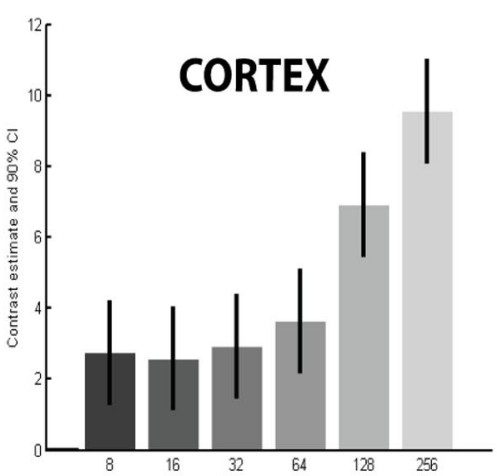

Stimulus Rate $\mathrm{Hz}$

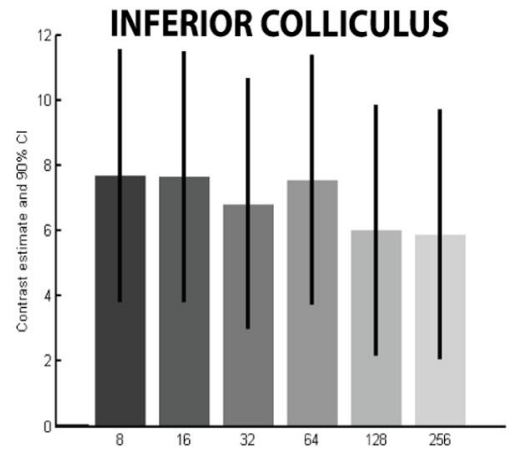

Stimulus Rate $\mathrm{Hz}$
Human

A

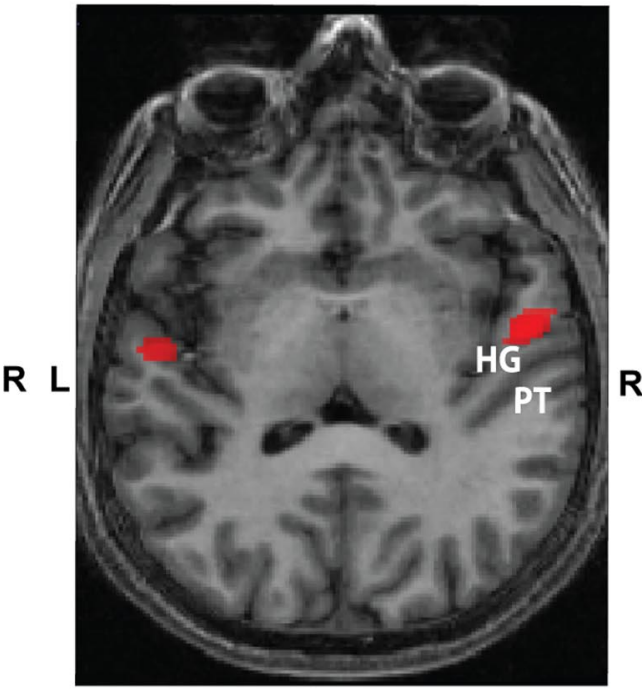

$\mathbf{P}$

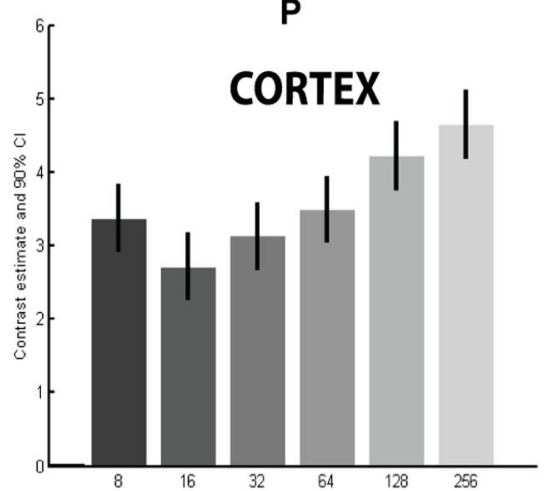

Stimulus Rate $\mathrm{Hz}$

INFERIOR COLLICULUS

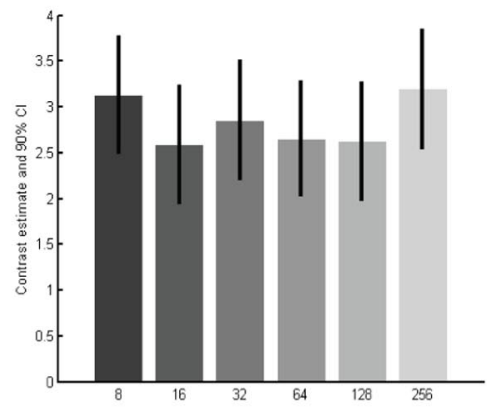

Stimulus Rate $\mathrm{Hz}$

Figure 1. Left column, Single-subject macaque BOLD data acquired at 4.7T are shown rendered onto a tilted axial section through the superior temporal plane (top). The macaque auditory core and belt areas defined by tonotopic mapping are outlined in blue and $A 1$ is marked on the right. The BOLD contrast shown is between regular-interval noise at rates above and below the lower limit of pitch in humans. Maxima are demonstrated anterolateral to A1 on either side. The histograms show no clear effect of stimulus rate on B0LD response in the inferior colliculus within the ascending auditory pathway, while mean responses in the cortical maxima increase from $32 \mathrm{~Hz}$ (close to the lower limit of pitch in humans). Data acquired by S. Baumann. Right column, Single-subject human BOLD data acquired at 3T are shown rendered onto a tilted axial section through the superior temporal plane (top). Heschl's Gyrus (HG) runs anterolaterally within the plane and the PT is situated behind it. The BOLD contrast shown is between regular-interval noise at rates above and below the lower limit of pitch in humans. No effect of rate on BOLD response is shown in the inferior colliculus while BOLD increases as a function of stimulus rate from $32 \mathrm{~Hz}$. Data acquired by T. Overath.

of a sound, although it is possible to control for this to some extent (Puschmann et al., 2010).

The studies above were all based on categorical comparison of pitch and control stimuli. Parametric designs, in which pitch strength is manipulated by continuous variation of the stimulus, provide a powerful way of seeking responses from putative pitch areas, which would be predicted to increase as a function of any accompanying change in pitch salience. A recent detailed study 


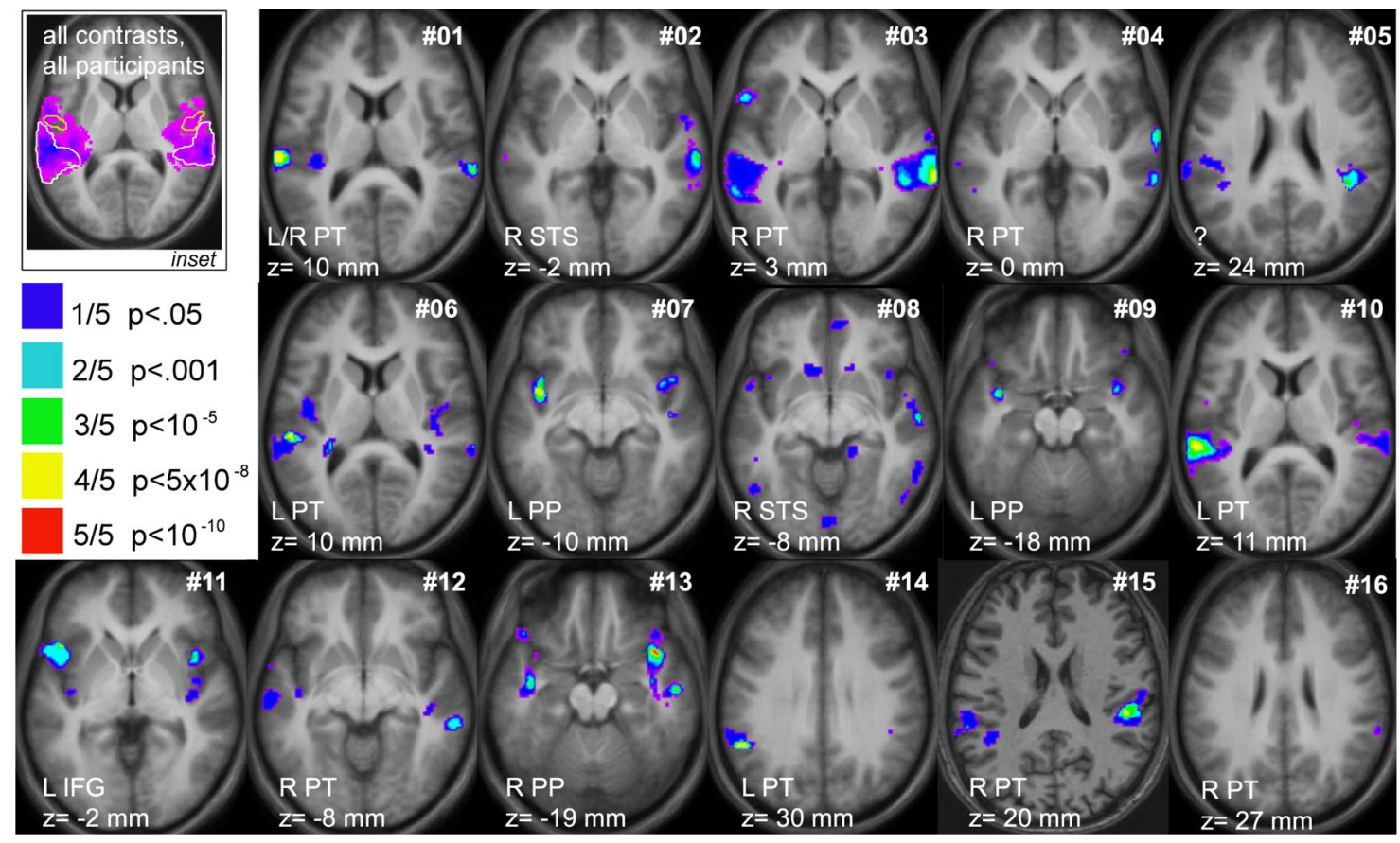

Figure 2. Individual subject analysis of fMRI data originally reported by Hall and Plack (2009). The inset shows group-averaged activity for all five pitch-evoking stimuli which encompasses HG (outlined in yellow) and PT (outlined in white). See the text for further explanations of the stimuli. The main panel illustrates those brain regions where there was most consistent pitch-related activity across the five pitch contrasts (i.e., pitch - noise control), within an individual subject. The color scale indicates the degree of consistency, with the associated probability of these spatially overlapping responses occurring by chance (all at $p<0.05$ ). Plane of each axial section is given in MNI (Montreal Neurological Institute) coordinates (mm), with the group-averaged activity represented at $z=10 \mathrm{~mm}$. PP, Planum polare; STS, superior temporal sulcus; IFG, inferior frontal gyrus).

(Barker et al., 2011), however, in which stimulus regularity and the associated pitch strength were varied continuously, did not show such a relationship in any area. Parametric stimulus manipulations also allow a critical test for a pitch area, which is to respond only when the stimulus parameters for sounds are in the pitch range. Figure 1 shows illustrative macaque and human data. In both species, activation is shown in the lateral superior temporal plane for the contrast between RIN with repetition rates above and below the lower limit of pitch in humans $(\sim 30 \mathrm{~Hz}$; Pressnitzer et al., 2001). The histograms show blood oxygenation level-dependent (BOLD) data as a function of repetition rate, with little change in the inferior colliculus, but responses in both species in the lateral cortical area that increase above the human lower limit of pitch.

Another suggested property of a pitch mechanism is that it should show pitch constancy: the same response to a given pitch value and strength regardless of the particular associated stimulus. This was first addressed (Hall and Plack, 2009) in a study using seven different stimuli, each with different spectral and temporal characteristics: pure tone, resolved and unresolved harmonic complex tones, a wideband harmonic-complex tone, a binaural pitch stimulus (Huggins pitch), and two types of RIN were all presented to each of 16 subjects. RIN produced responses in lateral HG in good agreement with previous studies. However, a different pattern of activation was reported for the other five pitch-evoking stimuli, with PT most consistently activated across the group. Moreover, the data showed similar distributions of neural activation for pure tones, resolved and unresolved harmonic complex tones, wideband harmonic-complex tones, and the binaural pitch despite their acoustic differences.

The categorical experiments using single stimuli and the experiments with multiple pitch stimuli assume pure insertion (Friston et al., 1996): a substrate for pitch representation that is independent of the perceptual and cognitive context. Experimental manipulation of pitch context has demonstrated that activity in primary cortex during pitch analysis is sensitive to context, while PT activation is independent of it (Garcia et al., 2010), consistent with the establishment of an invariant representation for pitch beyond primary cortex. Both forms of pitch analysis can be construed as levels within a hierarchical or heterarchical pitch system. Such organization can be examined in modeling studies using techniques similar to those used to model electrical data by Kumar and Schonwiesner (2012).

Many human studies employ group-level statistical inference and passive listening. These studies have the potential to overlook pitch-associated mechanisms that occur in functional areas that are anatomically variable between subjects or related to cognitive strategies that might vary between subjects. Figure 2 shows group data from Hall and Plack (2009) in addition to individual maps of pitch constancy. Ten subjects responded to at least four of the pitch contrasts within the same brain region, a condition with a probability of $<5 \times$ $10^{-8}$, supporting a role for this region in pitch coding. However, the location of this site varied a great deal from listener to listener; nine sites in the PT, three sites in the planum polare, two in the superior temporal sulcus, and in one subject, the inferior frontal gyrus. The functional role of these different regions could be examined using studies that systematically manipulate the pitch listening task in a within-subjects design.

The experiments above all use external stimuli. A pitch mechanism should be active during the perception of pitch regardless of whether any stimulus is present. Illusory pitch as an aftereffect has been examined using magnetoencephalography (Hoke et al., 1996), but not fMRI. Illusions and contextual effects that change pitch value or salience have not been exploited in fMRI studies to date but will provide a further test of the concept of a perceptual 
pitch center. A number of studies have addressed musical experience without external stimuli at the level of pitch sequences in studies of musical imagery (Zatorre and Halpern, 2005).

\section{Pitch sequences and timbre}

Most pitch studies have assessed sequences in which the pitch was fixed, but natural stimuli including vocalizations and music usually contain pitch variation across sequences or in the form of glides. In passive listening experiments, the effect of varying pitch in sequences shows more distributed representation in the superior temporal lobe than that corresponding to a constant pitch. Studies show bilateral activity in the anterior temporal lobes and the posterior part of the superior temporal gyrus (Patterson et al., 2002; Warren and Griffiths, 2003). Pitch-sequence studies in which there is an active listening task also engage the inferior lateral frontal lobe (Overath et al., 2007). Such frontal mechanisms in the right hemisphere have previously been emphasized as a substrate for pitch working memory (Zatorre et al., 1994).

Pitch stimuli are always associated with timbre: a distinct perceptual quality. A detailed discussion of timbre is beyond the scope of this review (see Griffiths et al., 2009), although behavioral evidence suggests an interdependence of pitch and timbre perception (Moore and Glasberg, 1990; Krumhansl and Iverson, 1992; see also Oxenham, 2012). A number of fMRI experiments in which timbral dimensions are modified independently of pitch point to overlapping early substrates in auditory cortex in HG and PT (Warren et al., 2005; Overath et al., 2008, 2010), in addition to the engagement of remote areas such as the superior temporal sulcus. The early overlapping substrates for pitch and timbre in HG and PT might in future be disambiguated using pattern analysis as below.

\section{Beyond conventional pitch mapping}

Multivariate pattern analysis allows the discrimination of responses to stimuli with different characteristics that cannot be discriminated using conventional fMRI analyses using massunivariate statistics (Haynes and Rees, 2006). A recent study (Staeren et al., 2009) examined the effect of pitch variation in natural sounds. Distinct patterns of response to different pitch values were demonstrated in the region spanning lateral $\mathrm{HG}$ and anterolateral PT. The result is consistent with the earlier studies using conventional fMRI analysis. Another way of examining responses within areas that are not shown by conventional analysis uses the phenomenon of repetition suppression: decrease in BOLD activity as a function of stimulus repetition. The technique allows a search for sensory coding mechanisms in neuronal populations independent of context. Models for the phenomenon (Grill-Spector et al., 2006) include adaptation within a defined population of neurons, a decrease in the pool of neurons from which a response occurs, and alteration in the response time course. The technique allows the search for suppression of responses to repeated pitch in a subpopulation of neurons regardless of the stimulus with which the pitch is associated, another predicted behavior of a pitch mechanism (Baumann et al., 2011).

\section{Future directions}

The human experiments above implicate regions lateral and posterior to primary auditory cortex in pitch representation, with ongoing debate about precise position. The data suggest a role for this area in pitch analysis based on experiments with different pitch-associated stimuli that might undergo initial sensory analysis in primary cortex. Preliminary data suggest that a similar pitch region lateral to primary cortex exists in the macaque, so that we are now in a position to establish a primate model for pitch perception using paradigms that are comparable to those used in humans. In addition to establishing regional organization, the macaque work will allow identification of key areas within the network for neurophysiology to further establish neuronal mechanisms for the abstraction and use of pitch.

Human fMRI is particularly suitable for examining the distributed processing circuit supporting pitch cognition, which has not been possible in previous studies based on passive listening. The roles of lateral and posterior portions of the superior temporal plane and their remote connections in active pitch listening, including selective attention, working memory, and object categorization, require further definition.

\section{References}

Barker D, Plack CJ, Hall DA (2011) Human auditory cortical responses to pitch and to pitch strength. Neuroreport 22:111-115. CrossRef Medline

Barker D, Plack CJ, Hall DA (2012) Reexamining the evidence for a pitchsensitive region: a human fMRI study using iterated ripple noise. Cereb Cortex 22:745-753. CrossRef Medline

Baumann S, Kumar S, Overath T, Griffiths TD (2011) Repetition suppression for pitch using fMRI. Soc Neurosci Abstr 37:171.02.

Da Costa S, van der Zwaag W, Marques JP, Frackowiak RS, Clarke S, Saenz M (2011) Human primary auditory cortex follows the shape of Heschl's gyrus. J Neurosci 31:14067-14075. CrossRef Medline

Formisano E, Kim DS, Di Salle F, van de Moortele PF, Ugurbil K, Goebel R (2003) Mirror-symmetric tonotopic maps in human primary auditory cortex. Neuron 40:859-869. CrossRef Medline

Friston KJ, Price CJ, Fletcher P, Moore C, Frackowiak RSJ, Dolan RJ (1996) The trouble with cognitive subtraction. Neuroimage 4:97-104. CrossRef Medline

Garcia D, Hall DA, Plack CJ (2010) The effect of stimulus context on pitch representations in the human auditory cortex. Neuroimage 51:808-816. CrossRef Medline

Griffiths TD, Kumar S, Von Kriegstein K, Overath T, Stephan KE, Friston KJ (2009) Auditory object analysis. In: The cognitive neurosciences (Gazzaniga MS, ed), pp 367-381. Cambridge, MA: MIT.

Griffiths TD, Kumar S, Sedley W, Nourski KV, Kawasaki H, Oya H, Patterson RD, Brugge JF, Howard MA (2010) Direct recordings of pitch responses from human auditory cortex. Curr Biol 20:1128-1132. CrossRef Medline

Grill-Spector K, Henson R, Martin A (2006) Repetition and the brain: neural models of stimulus-specific effects. Trends Cogn Sci 10:14-23. CrossRef Medline

Hall DA, Plack CJ (2007) The human 'pitch center' responds differently to iterated noise and Huggins pitch. Neuroreport 18:323-327. CrossRef Medline

Hall DA, Plack CJ (2009) Pitch processing sites in the human auditory brain. Cereb Cortex 19:576-585. CrossRef Medline

Hall DA, Edmondson-Jones AM, Fridriksson J (2006) Periodicity and frequency coding in human auditory cortex. Eur J Neurosci 24:3601-3610. CrossRef Medline

Haynes JD, Rees G (2006) Decoding mental states from brain activity in humans. Nat Rev Neurosci 7:523-534. CrossRef Medline

Hoke ES, Hoke M, Ross B (1996) Neurophysiological correlate of the auditory after-image ("Zwicker tone"). Audiol Neurootol 1:161-174. CrossRef Medline

Humphries C, Liebenthal E, Binder JR (2010) Tonotopic organization of human auditory cortex. Neuroimage 50:1202-1211. CrossRef Medline

Krumhansl CL, Iverson P (1992) Perceptual interactions between musical pitch and timbre. J Exp Psychol Hum Percept Perform 18:739-751. CrossRef Medline

Kumar S, Schönwiesner M (2012) Mapping human pitch representation in a distributed system using depth-electrode recordings and modeling. J Neurosci 32:13348-13351. CrossRef

Langers DR, van Dijk P (2011) Mapping the tonotopic organization in human auditory cortex with minimally salient acoustic stimulation. Cereb Cortex. Advance online publication. Retrieved August 24, 2012. CrossRef

Logothetis NK, Pauls J, Augath M, Trinath T, Oeltermann A (2001) Neurophysiological investigation of the basis of the fMRI signal. Nature 412: 150-157. CrossRef Medline 
Moore BC, Glasberg BR (1990) Frequency discrimination of complex tones with overlapping and non-overlapping harmonics. J Acoust Soc Am 87: 2163-2177. CrossRef Medline

Overath T, Cusack R, Kumar S, von Kriegstein K, Warren JD, Grube M, Carlyon RP, Griffiths TD (2007) An information theoretic characterisation of auditory encoding. PLoS Biol 5:e288. CrossRef Medline

Overath T, Kumar S, von Kriegstein K, Griffiths TD (2008) Encoding of spectral correlation over time in auditory cortex. J Neurosci 28:13268-13273. CrossRef Medline

Overath T, Kumar S, Stewart L, von Kriegstein K, Cusack R, Rees A, Griffiths TD (2010) Cortical mechanisms for the segregation and representation of acoustic textures. J Neurosci 30:2070-2076. CrossRef Medline

Oxenham AJ (2012) Pitch perception. J Neurosci 32:13335-13338. CrossRef

Patterson RD, Uppenkamp S, Johnsrude IS, Griffiths TD (2002) The processing of temporal pitch and melody information in auditory cortex. Neuron 36:767-776. CrossRef Medline

Penagos H, Melcher JR, Oxenham AJ (2004) A neural representation of pitch salience in nonprimary human auditory cortex revealed with functional magnetic resonance imaging. J Neurosci 24:6810-6815. CrossRef Medline

Petkov CI, Kayser C, Augath M, Logothetis NK (2006) Functional imaging reveals numerous fields in the monkey auditory cortex. PLoS Biol 4:e215. CrossRef Medline
Pressnitzer D, Patterson RD, Krumbholz K (2001) The lower limit of melodic pitch. J Acoust Soc Am 109:2074-2084. CrossRef Medline

Puschmann S, Uppenkamp S, Kollmeier B, Thiel CM (2010) Dichotic pitch activates pitch processing centre in Heschl's gyrus. Neuroimage 49:1641-1649. Medline

Staeren N, Renvall H, De Martino F, Goebel R, Formisano E (2009) Sound categories are represented as distributed patterns in the human auditory cortex. Curr Biol 19:498-502. CrossRef Medline

Talavage TM, Sereno MI, Melcher JR, Ledden PJ, Rosen BR, Dale AM (2004) Tonotopic organization in human auditory cortex revealed by progressions of frequency sensitivity. J Neurophysiol 91:1282-1296. Medline

Warren JD, Griffiths TD (2003) Distinct mechanisms for processing spatial sequences and pitch sequences in the human auditory brain. J Neurosci 23:5799-5804. Medline

Warren JD, Jennings AR, Griffiths TD (2005) Analysis of the spectral envelope of sounds by the human brain. Neuroimage 24:1052-1057. CrossRef Medline

Woods DL, Stecker GC, Rinne T, Herron TJ, Cate AD, Yund EW, Liao I, Kang X (2009) Functional maps of human auditory cortex: effects of acoustic features and Attention. PLoS One 4:e5183. CrossRef Medline

Zatorre RJ, Halpern AR (2005) Mental concerts: musical imagery and auditory cortex. Neuron 47:9-12. CrossRef Medline

Zatorre RJ, Evans AC, Meyer E (1994) Neural mechanisms underlying melodic perception and memory for pitch. J Neurosci 14:1908-1919. Medline 